\title{
Relativistic Atomic Structure and Spectral Data Calculations for O-Like Tungsten Ion
}

\author{
S.M. HAMASHA \\ Department of Physics, the Hashemite University, P.O. Box 150459, Zarqa, Jordan \\ Received: 20.01.2020 \& Accepted: 25.08.2020
}

Doi: $10.12693 /$ APhysPolA.138.770

*e-mail: safeia@hu.edu.jo

\begin{abstract}
Accurate atomic data of tungsten is of great importance for plasma research groups including the International Thermonuclear Experimental Reactor because its facing material is made of tungsten (W) which displays complex spectra. There is a wealth of experimental and theoretical atomic data for most tungsten ions but published atomic data of $\mathrm{W}^{66+}(\mathrm{O}-$ like $\mathrm{W})$ is scarce. This paper presents a large scale accurate atomic structure and spectral data for this ion in order to fill out this gap. The presented data is calculated by employing two methods: the relativistic multi-configuration interaction approximations and the multireference many-body perturbation theory. Transitions between the excited and ground states of $2 l-n l^{\prime}$ are considered in the calculations, where $n=3,4,5$ and $6, l$ is $s$ or $p$ states and $l^{\prime}$ is the proper corresponding orbital angular momentum of shell $n$. The correlation effects, the relativistic and QED effects are included in the calculations. Detailed atomic data includes energy levels, weighted oscillator strengths and transition rates for electric-dipole $\left(E_{1}\right)$, electric-quadrupole $\left(E_{2}\right)$, magneticdipole $\left(M_{1}\right)$ and magnetic-quadrupole $\left(M_{2}\right)$ transitions. The generated data has been used to construct synthetic spectra for $E_{1}, E_{2}, M_{1}$ and $M_{2}$ transitions. The comparison of the procured atomic data with the published experimental and theoretical data of $\mathrm{W}^{66+}$ demonstrates good agreement. Findings of this paper are essential for W-plasma diagnostics and fusion research, as well as for other applications of highly ionized tungsten ions.
\end{abstract}

topics: atomic data, plasma spectroscopy, transition probabilities, multipole transitions

\section{Introduction}

Tungsten (W) presents remarkable metallic properties such as high density, corrosion resistance, high melting point and low vapor pressure [1] which make it an excellent facing material for tokamak and fusion devices. The facing material of the International Thermonuclear Experimental Reactor (ITER), which is believed to be the next step in the experimental fusion devices, is made of tungsten reinforced composite with beryllium and carbonfiber [2]. As a result, tungsten appears as impurities in produced plasma of those experiments. Many ionization stages of tungsten radiate strongly in the spectral range from VUV to soft X-ray [3] which suggests that a significant energy fraction in the plasma is shattered via radiation emitted by tungsten ions. Therefore, it is important to compile reliable database of atomic structure, multipole transitions and synthetic spectra for tungsten at wide temperature ranges and ionization stages in order to facilitate interpreting and validating experimental observations of the corresponding plasma.

There are currently vast experimental and theoretical efforts to produce high ionization states in tungsten plasma in order to investigate tungsten ions and their belongings [4-9]. The published results include measurements of transition energies with poor precision $[4,5]$ and improved precision upon measuring tungsten transitions emitted from laser-produced plasmas $[6-9]$. The electron beam ion traps (EBIT-I and EBIT-II) at the Lawrence Livermore National Laboratory (LLNL), which were built to study the spectroscopy of highly charged ions produced at well-controlled conditions, greatly improved the quality of measuring atomic properties of tungsten ions. Several studies of X-ray and EUV spectra of tungsten ions at different charges have already been carried out [10-16]. Tokamak plasma experiments have also produced several ionization stages of tungsten ions $[17-20]$.

Theoretical calculations for ionization stages and atomic processes for tungsten ions in plasma attract several research groups. Previous calculations focused on the structure and spectral atomic data, as well as modeling experimental results [21-28]. Kramida and Shirai compiled theoretical and experimental data of energy levels and spectral lines for multiply ionized $\mathrm{W}^{2+}$ through $\mathrm{W}^{73+}$ ions [29]. However, forbidden transitions lines, which are linked to plasma diagnostics of electrons density and temperature, were not subject to comparable research efforts [30-33]. 
The aim of this paper is to provide largescale atomic data calculations for energy levels and spectra of allowed and forbidden transitions of O-like $\mathrm{W}$ ion to be used in future laboratory plasma studies and fusion research. Two calculation methods are applied: the relativistic configuration interaction (RCI) method of the flexible atomic code (FAC) [34] and the multireference many-body perturbation theory (MR-MBPT) [35] which is close to the method of the multireference Moller-Plesset perturbation theory (MR-MP) [36]. The RCI method yields quantitative predictions which perfectly agree with experimental findings, including for example those published in [37, 38]. The MR-MBPT method generates accurate atomic data for many electron systems [35]. Large-scale relativistic atomic calculations are employed between ground state shell to higher excited states, i.e., from $2 s^{2} 2 p^{4}$ to $2 s^{2} 2 p^{3} n l, 2 s 2 p^{5}$ to $2 s 2 p^{4} n l$ or $2 p^{6}$ to $2 p^{5} n l$, where $n=3,4,5$ and 6 , and $l$ is the proper angular momentum of $n$ shells. The inner shell transitions $\Delta n=0$ for $n=2$ are not included in the current calculations because their transitions are in a different spectral region, the UV region.

The RCI method yields accurate data because it uses the standard Coulomb-Dirac Hamiltonian that includes the spin-orbit interaction, mass-shift and other leading relativistic effects. In order to account for finite effects of the nuclear size, nuclear potential is assumed to be exerted by a hard sphere. Some quantum electrodynamic QED corrections are also included in the Hamiltonian. Correlation and relativistic effects are calculated by the following DiracFock method. Correlation, relativistic and QED effects play an important role in the atomic structure of many electron systems and their spectral properties. The multi-configuration expansion includes correlation effects of all orders. In practice, effects of electron correlation on the fine structures and transitions are more important than QED. Therefore, high orders of QED corrections, such as retardation and nuclear recoil, are not seen in the RCI of the FAC. The MR-MBPT calculation method is based on the relativistic multi-configuration interaction approximations. It is advantageous over the RCI method because it involves additional corrections including QED effects and vacuum polarization which are calculated by applying the second order perturbation theory. However, the second order perturbation theory makes the matrices of the Hamiltonian very large when dealing with a high number of electrons in open shells or high shell numbers which may lead to a data jam that terminates the run abruptly, without accomplishing the required calculations.

Energy levels of O-like $\mathrm{W}$ presented in this paper are calculated by the RCI and the MR-MBPT methods. The latter method is also used to produce multipole transitions data that is essential for low density and hot dense tungsten plasma diagnostics. The produced data, which includes energy levels, transition rates, oscillator strengths and wavelengths for electric dipole $\left(E_{1}\right)$, electric quadrupole $\left(E_{2}\right)$, magnetic dipole $\left(M_{1}\right)$ and magnetic quadrupole $\left(M_{2}\right)$, is presented in a handy format and is used to construct synthetic spectra for multipole transitions.

\section{Theoretical methods and computational procedure}

\subsection{Multireference many-body perturbation theory}

The proposed method starts from the RayleighSchrödinger perturbation theory for a multiconfigurational model space. Detailed derivation and formulation can be found in [38], while a brief overview is presented below. The Schrödinger equation of a many-electron system is solved by perturbation expansion. One starts from the eigenproblem

$$
H_{\mathrm{DCB}} \varphi_{I}=E_{I} \varphi_{I},
$$

where the Dirac-Coulomb-Breit Hamiltonian $H_{\mathrm{DCB}}$ for particle $i$ at position $\boldsymbol{r}_{i}$ is given as

$$
H_{\mathrm{DCB}}=\sum_{i}\left(H_{d}(i)-\frac{Z}{r_{i}}\right)+\sum_{i<j}\left(\frac{1}{r_{i j}}+B_{i j}\right) \text {. }
$$

Here, $H_{d}(i)$ is the free electron Dirac Hamiltonian, $r_{i}$ is the electron radial coordinate and $\boldsymbol{r}_{i j}$ is the vector separation between the electrons $i$ and $j$. The Breit interaction $B_{i j}$ is given as

$$
B_{i j}=-\frac{\boldsymbol{\alpha}_{i} \boldsymbol{\alpha}_{j}}{2 r_{i j}}+\frac{\left(\boldsymbol{\alpha}_{i} \boldsymbol{r}_{j}\right)\left(\boldsymbol{\alpha}_{j} \boldsymbol{r}_{i}\right)}{2 r_{i j} r_{i j}^{2}}
$$

and $\boldsymbol{\alpha}_{i}$ is the matrix vector of the Pauli spin matrices $\sigma_{i}$. The Breit Hamiltonian (3) is composed of several energy operators for electrons in electric and magnetic fields

$B_{i j}=\hat{H}_{0}+\hat{H}_{1}+\hat{H}_{2}+\hat{H}_{3}+\hat{H}_{4}+\hat{H}_{5}+\hat{H}_{6}$,

where $\hat{H}_{0}=\sum_{i} \frac{\boldsymbol{p}_{i}^{2}}{2 m_{i}}+V_{0}$ is the non-relativistic Hamiltonian, and $\hat{H}_{1}=-\sum_{i} \boldsymbol{p}_{i}^{4} /\left(8 c^{2} m_{i}^{3}\right)$ is the relativistic energy correction to the Hamiltonian. Next,

$\hat{H}_{2}=-\sum_{i>j} \frac{q_{i} q_{j}}{2 r_{i j} m_{i} m_{j} c^{2}}\left(\boldsymbol{p}_{i} \boldsymbol{p}_{j}+\frac{\left(\boldsymbol{r}_{i j} \boldsymbol{p}_{i}\right)\left(\boldsymbol{r}_{i j} \boldsymbol{p}_{j}\right)}{r_{i j}^{2}}\right)$

is the interaction between the magnetic dipole moments of the particles of charges $q_{i}$ and $q_{j}$ which partly accounts for retardation and is called the orbit-orbit interaction. In turn, interaction between the spin magnetic moment and the orbital magnetic moments (the spin-orbit interaction) is described with

$\hat{H}_{3}=\frac{\mu_{\mathrm{B}}}{c} \sum_{i} \boldsymbol{s}_{i}\left(\frac{\boldsymbol{\varepsilon}\left(\boldsymbol{r}_{i}\right) \times \boldsymbol{p}_{i}}{m_{i}}+\sum_{j>i} \frac{2 q_{i}}{m_{i} r_{i j}^{3}} \boldsymbol{r}_{i j} \times \boldsymbol{p}_{j}\right)$,

where $\varepsilon\left(\boldsymbol{r}_{i}\right)$ is the electric field at the particle's position. 
TABLE I

Distribution of the total angular momentum $(J)$ over even and odd parities of energy levels of O-like W (the total number of energy levels is 1546).

\begin{tabular}{c|c|c|c|c|c|c|c|c|c}
\hline \hline$J$ & 0 & 1 & 2 & 3 & 4 & 5 & 6 & 7 & 8 \\
\hline even & 62 & 151 & 185 & 158 & 112 & 66 & 29 & 10 & 1 \\
odd & 59 & 153 & 182 & 159 & 112 & 65 & 31 & 9 & 2
\end{tabular}

Next, $\hat{H}_{4}=\frac{\mathrm{i} h}{8 \pi c^{2}} \sum_{i} \frac{q_{i}}{m_{i}^{3}} \boldsymbol{p}_{i} \varepsilon\left(\boldsymbol{r}_{i}\right)$ is the Darwin term from the Dirac theory and $\hat{H}_{5}$ is the spin-spin interaction, i.e.,

$$
\begin{gathered}
\hat{H}_{5}=-\frac{32 \mu_{B}^{2} \pi}{3} \sum_{i>j}\left[\boldsymbol{s}_{i} \boldsymbol{s}_{j} \delta\left(\boldsymbol{r}_{i j}\right)+\frac{1}{r_{i j}^{3}} \boldsymbol{s}_{i} \boldsymbol{s}_{j}\right. \\
\left.-\frac{3\left(\boldsymbol{s}_{i} \boldsymbol{r}_{i j}\right)\left(\boldsymbol{s}_{j} \boldsymbol{r}_{i j}\right)}{r_{i j}^{5}}\right] .
\end{gathered}
$$

The first term of $\hat{H}_{5}$ is called the contact interaction and the second one is the dipole-dipole interaction. The last part of the Dirac-Coulomb-Breit interaction is $\hat{H}_{6}$, describing the interaction between spin and orbital magnetic moments with an external magnetic field $\boldsymbol{H}$ :

$$
\hat{H}_{6}=2 \mu_{B} \sum_{i} \boldsymbol{H}\left(\boldsymbol{r}_{i}\right) \boldsymbol{s}_{i}+\frac{q_{i}}{m_{i} c} \boldsymbol{A}\left(\boldsymbol{r}_{i}\right) \boldsymbol{p}_{i} .
$$

The fundamental step of the MR-MBPT method is to divide the full Hamiltonian Hilbert space into two model spaces $\mathcal{M}$ and $\mathcal{N}$ - both spaces are orthogonal. The non-Hermitian effective Hamiltonian is contained in $\mathcal{M}$ model space, while $\mathcal{N}$ contains the perturbation expansion. The energy levels are the eigenvalues of the effective Hamiltonian. The multi-configuration interaction effects within the model space are fully included and interactions between $\mathcal{M}$ and $\mathcal{N}$ are taken into account with the perturbation method.

We may also write the Hamiltonian as

$$
H_{\mathrm{DCB}}=H_{0}+H^{\prime} \text {, }
$$

where

$$
H_{0}=\sum_{i} H_{d}\left(\boldsymbol{r}_{i}\right)+U\left(\boldsymbol{r}_{i}\right)
$$

and

$$
H^{\prime}=\sum_{i}\left[-U\left(\boldsymbol{r}_{i}\right)+\frac{Z}{r_{i}}+\sum_{i<j} \frac{1}{r_{i j}}+B_{i j}\right] .
$$

The potential $U\left(\boldsymbol{r}_{i}\right)$ includes the screening effect of all electrons. It is approximated by a local central potential derived from Dirac-Fock-Slater selfconsistent field calculations. It should be chosen properly to make the perturbation potential $H^{\prime}$ to be as small as possible. The model space $\mathcal{M}$ contains all configurations of O-like $\mathrm{W}$ ion in the shell $n=2$. The $\mathcal{N}$ space contains all electronic configurations of the excited electrons of shell number $n=3,4$ or 5 or 6 , as listed in Table I. Strong configuration mixing among the highly excited levels is obtained by applying the multireference configuration interaction approximations [34].
Values of the energy levels are attained by solving a generalized eigenvalue problem. The total energy $E_{I}$ is the sum of the eigenvalues of the multi-configuration Dirac-Coulomb-Breit Hamiltonian matrix $\left(E_{I}^{C I}\right)$ and the second order perturbation corrections due to QED corrections in the space $\mathcal{N}\left(E_{I}^{(2)}\right)$, where CI stands for the configuration interaction approximations.

\subsection{Relativistic multi-configuration interaction method}

The RCI method starts from the Dirac equations. In sight of the electron screening of possible configurations, an imitation mean configuration is constructed for O-like $\mathrm{W}$ ion with a fractional occupation number. The bound states systems are calculated by configuration mixing approximations with a convenient specification of a mixing scheme. A modified iteration for the self-consistent DiracFock-Slater potential is used to derive the local central potential. The radial orbitals for the constructed basis states are, then, derived by the consequent local central potential. A correction procedure is applied to reduce errors in energy levels through the diagonalization of the relativistic Hamiltonian.

The atomic ion relativistic Hamiltonian is the sum over the Dirac Hamiltonian of a single electron due to an electron-electron interaction and nuclear charge contribution potentials. The electronelectron interaction contains the bound electrons averaged potential and the exchange interactions local approximations. A self-consistent iteration is constructed to solve the Dirac coupled equations, wherein the radial orbital from the prior step is used to derive the potential. The small component of the Dirac coupled equations is eliminated to convert the coupled equations to only one differential equation after performing some appropriate transformations. The standard Numerov method is used to solve the produced differential equation numerically. The theory of the FAC is detailed in [33].

The single multipole approximation method is used to calculate the radiative transition rates $A_{f i}$. This method uses the second quantization with the help of the Racah algebra, thus one applies the expression $[39,40]$ :

$$
g_{f} A_{f i}=\frac{\alpha^{3} \omega^{3}}{2 L}(\alpha \omega)^{2 L-2} S_{i f},
$$

where $S_{i f}$ is the generalized strength of the transition line, given as $S_{f i}=\left|\left\langle\varphi_{f}\left|O_{M}^{L}\right| \varphi_{i}\right\rangle\right|^{2}$ with the multipole operator $O_{M}^{L}$ and $\omega=E_{f}-E_{i}$ as the transition energy. The rates of forbidden transitions $\left(A\right.$-values in $\left.\mathrm{s}^{-1}\right)$ in terms of line strength $S_{i f}$ (arb.u. - arbitrary units) and the wavelength $\lambda$

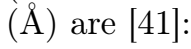

$$
A_{f i}^{E_{1}}=\frac{2.0613 \times 10^{18}}{(2 J+1) \lambda^{3}} S_{i f}^{E_{1}},
$$




$$
\begin{aligned}
& A_{f i}^{M_{1}}=\frac{2.6973 \times 10^{13}}{(2 J+1) \lambda^{3}} S_{i f}^{M_{1}} \\
& A_{f i}^{E_{2}}=\frac{1.1199 \times 10^{18}}{(2 J+1) \lambda^{5}} S_{i f}^{E_{2}}, \\
& A_{f i}^{M_{2}}=\frac{1.4909 \times 10^{13}}{(2 J+1) \lambda^{5}} S_{i f}^{M_{2}},
\end{aligned}
$$

where $E_{1}$ is the electric dipole, $E_{2}$ is the electric quadrupole, $M_{1}$ is the magnetic dipole and $M_{2}$ is the magnetic quadrupole.

\section{Results and discussion}

\subsection{Energy levels of $\mathrm{W}^{66+}$}

Table I presents the electronic configurations of O-like W which have been considered in the calculations. They are grouped and listed by the shell number. There are 1546 generated energy levels of even and odd parities for shell numbers $n=2,3,4$, 5 and 6 . They are distributed as in Table SI in the supplementary material [42].

A detailed atomic structure is acquired through the diagonalization of the system Hamiltonian. The atomic structure is calculated via the RCI and the MR-MBPT methods. The two calculation methods yield energy values with a pretty good agreement. The difference between the corresponding energy values calculated by the two methods is found to be in the range from -0.893 to $0.791 \mathrm{eV}$ for all energy levels of $n=2,3,4,5$ and 6 shells. The maximum percentage difference is about $0.011 \%$. These slight differences are likely caused by including QED effects of high orders in the MR-MBPT method, which seem to be of low significance in O-like $\mathrm{W}$ ion. The calculated data does not exhibit any mixing among states which implies that energy levels orderings are similar in the two calculation methods. Figure 1 shows the percentage differences distribution of the calculated

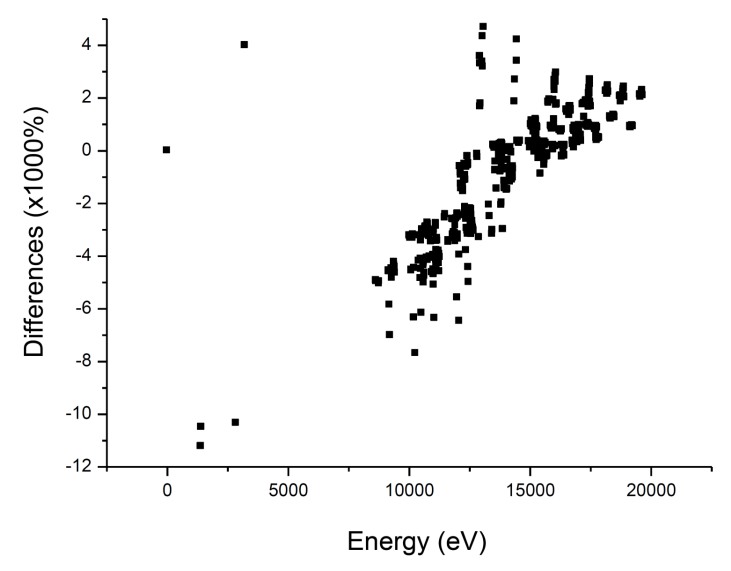

Fig. 1. Differences in percent between the calculated values of energy levels by the RCI method and the MR-MBPT method.
TABLE II

Comparison with the available published experimental energy levels of $\mathrm{W}^{66+}$.

\begin{tabular}{c|c|c|c|c}
\hline \hline $\begin{array}{c}\text { State } \\
\text { configuration }\end{array}$ & $J$ & $\begin{array}{c}E[\mathrm{eV}] \\
\text { from [37] }\end{array}$ & $E_{\mathrm{RCI}}[\mathrm{eV}]$ & $E_{\mathrm{MBPT}}[\mathrm{eV}]$ \\
\hline $2 s^{2} 2 p^{4}$ & & 0 & 0 & 0 \\
$2 s^{2} 2 p^{4}$ & 0 & 61.7 & 61.338 & 61.332 \\
$2 s 2 p^{5}$ & & 1806.0 & 1807.453 & 1807.098 \\
$2 s 2 p^{5}$ & 1 & 1890.8 & 1892.382 & 1891.884
\end{tabular}

energy values by RCI and MR-MBPT for shells from $n=2$ to $n=6$. In Table II, the calculated energy levels data demonstrates a good agreement with published experimental data produced by the electron beam ion trap [37]. A maximum percentage difference between the calculated and experimental data is $0.04 \%$.

\subsection{Optically allowed transitions of $\mathrm{W}^{66+}$}

The spectral atomic data is based only on the calculations by the MR-MBPT method, because it includes the QED corrections which are not available in the RCI method. Accordingly, only atomic data produced by the MR-MBPT method for the multipole transitions are presented in Table SII in the supplementary material [42]. The strong electric dipole transitions $E_{1}$ atomic data of O-like W, such as transition energies $\Delta E(\mathrm{eV})$, wavelengths $\lambda(\AA)$, radiative transition rates $A_{r}\left(\mathrm{~s}^{-1}\right)$ and weighted oscillator strengths $g h_{i j}$ is listed in Table II. Transitions in the complexes $n=2 l$ to $n^{\prime} l^{\prime}$, where $l$ is $s$ or $p, n^{\prime}=4,5,6$ and 7 , and $l^{\prime}$ is the proper angular momentum of $n^{\prime}$ shell, are considered. Configuration state functions which are listed in Table I are used in the calculations of the electric dipole transitions $E_{1}$ atomic data. The calculated $E_{1}$ values produce a very large number of lines, therefore, only strong lines for 2-3, 2-4, 2-5, and 2-6 transitions with $\mathrm{A}$-value $\geq 1.76 \times 10^{14} \mathrm{~s}^{-1}$ are listed in Table II. It is evident that $2 l-3 l^{\prime}$ transitions are the strongest. The electric dipole spectral range is situated in the wavelength range $0.75-1.44 \AA$. It is worth noting that closed subshells are not listed in the electronic configurations lists.

The comparison of $E_{1}$ transitions against the published experimental or theoretical data is only possible for a few transitions due to the scarcity of the published experimental and theoretical atomic data of multipole transitions for O-like W. Therefore, Table III presents the comparison between the findings of the current study against the published experimental values which were measured in the electron beam ion trap [43] along with calculated data by the FAC as well as theoretical transition energies that were calculated by two other methods: the multi-configuration Dirac-Fock method of GRASP92 code $[44,45]$ and the multi-configuration Dirac-Fock method of (MCDF) code [46]. 
TABLE III

Comparison of excitation energies $\Delta E[\mathrm{eV}]$ for some $\mathrm{W}^{66+}$ transitions with published experimental (exp.) and calculated data (cal.) in a given reference.

\begin{tabular}{|c|c|c|c|c|c|c|c|c|c|c|}
\hline \multirow[b]{2}{*}{ Line \# } & \multirow[b]{2}{*}{ Upper state } & \multirow[b]{2}{*}{$J_{\text {up }}$} & \multirow[b]{2}{*}{ Lower state } & \multirow[b]{2}{*}{$J_{\text {low }}$} & \multicolumn{6}{|c|}{$\Delta E[\mathrm{eV}]$} \\
\hline & & & & & $\begin{array}{c}\text { Exp. } \\
\text { Ref. [43] }\end{array}$ & $\begin{array}{c}\text { Cal. } \\
\text { Ref. [43] }\end{array}$ & \begin{tabular}{|c|} 
Cal. \\
Ref. [44]
\end{tabular} & $\begin{array}{c}\text { Cal. } \\
\text { Ref. [45] }\end{array}$ & (RCI) & $(\mathrm{MBPT})$ \\
\hline $\mathrm{O}-1$ & $3 s^{2} 3 p_{1 / 2}^{2} 3 p_{3 / 2} 3 s_{1 / 2}$ & 1 & $3 s_{1 / 2}^{2} 3 p_{1 / 2}^{2} 2 p_{3 / 2}^{2}$ & 0 & $8592.7 \pm 0.7$ & 8588.4 & 8590.09 & 8591.4 & 8589.454 & 8588.583 \\
\hline $\mathrm{O}-2$ & $3 s_{1 / 2} 3 p_{1 / 2}^{2} 3 p_{3 / 2}^{2} 3 s_{1 / 2}$ & 2 & $3 s_{1 / 2} 3 p_{1 / 2}^{2} 2 p_{3 / 2}^{3}$ & 2 & 1.0 & 8595.26 & 8595.15 & - & 30 & 597991 \\
\hline $\mathrm{O}-3$ & $3 s_{1 / 2} 3 p_{1 / 2}^{2} 3 p_{3 / 2}^{2} 3 s_{1 / 2}$ & 2 & $3 s_{1 / 2} 3 p_{1 / 2}^{2} 2 p_{3 / 2}^{3}$ & 1 & 8607.1 & 8604.21 & 8604.13 & - & 8604.643 & 860 \\
\hline $\mathrm{O}-4$ & $3 s^{2} 3 p_{1 / 2}^{2} 3 p_{3 / 2} 3 \mathrm{~s}_{1 / 2}$ & 2 & $3 s_{1 / 2}^{2} 3 p_{1 / 2}^{2} 2 p_{3 / 2}^{2}$ & 2 & $8644.5 \pm 0.6$ & 8640.19 & 8641.6 & 8643 & 8645.269 & 8645.475 \\
\hline O-5 & $3 s^{2} 3 p_{1 / 2}^{2} 3 p_{3 / 2} 3 s_{1 / 2}$ & 1 & $3 s_{1 / 2}^{2} 3 p_{1 / 2}^{2} 2 p_{3 / 2}^{2}$ & 2 & $8654.4 \pm 0.6$ & 8649.51 & 8650.82 & 8652.2 & 8654.158 & 8653.456 \\
\hline O-6 & $3 \mathrm{~s}^{2} 3 \mathrm{p}_{1 / 2}^{2} 3 \mathrm{p}_{3 / 2} 3 \mathrm{~d}_{5 / 2}$ & 2 & $3 \mathrm{~s}_{1 / 2}^{2} 3 \mathrm{p}_{1 / 2}^{2} 2 \mathrm{p}_{3 / 2}^{2}$ & 2 & - & 9396.9 & - & - & 9397.047 & 9397.46 \\
\hline $\mathrm{O}-7$ & $3 s^{2} 3 p_{1 / 2}^{2} 3 p_{3 / 2} 3 d_{5 / 2}$ & 1 & $3 s_{1 / 2}^{2} 3 p_{1 / 2}^{2} 2 p_{3 / 2}^{2}$ & 2 & $9413.6 \pm 1.2$ & 9413.02 & 9413.92 & - & 9413.261 & 9413.260 \\
\hline $\mathrm{O}-8$ & $3 s^{2} 3 p_{1 / 2} 3 p_{3 / 2}^{2} 3 d_{5 / 2}$ & 3 & $3 s_{1 / 2}^{2} 3 p_{1 / 2}^{2} 2 p_{3 / 2}^{2}$ & 2 & - & 2 1071 & - & - & 10712.6 & 10712.10 \\
\hline O-9 & $3 s^{2} 3 p_{1 / 2} 3 p_{3 / 2}^{2} 3 d_{5 / 2}$ & 2 & $3 s_{1 / 2}^{2} 3 p_{1 / 2}^{2} 2 p_{3 / 2}^{2}$ & 2 & - & 10714.94 & - & - & 10712.17 & 10715.51 \\
\hline $\mathrm{O}-10$ & $3 s^{2} 3 p_{1 / 2} 3 p_{3 / 2}^{2} 3 d_{5 / 2}$ & 1 & $3 s_{1 / 2}^{2} 3 p_{1 / 2}^{2} 2 p_{3 / 2}^{2}$ & 2 & - & 10721.66 & - & - & 10722.27 & 10722.27 \\
\hline
\end{tabular}

The experimental lines appearing in the comparison Table III were previously measured by the electron beam ion trap by Beiersdorfer et al. who labeled their predicted O-like $\mathrm{W}$ lines as $\mathrm{O}-0, \mathrm{O}-1$, ..., O-10 [43]. Some of these lines were identified experimentally but the rest were predicted theoretically. The first theoretically predicted line is O-0. Its transition energy is $8400.61 \mathrm{eV}$. In 2012, Beiersdorfer et al. pointed out that the O-0 line could not be experimentally identified but their calculations predicted the strength of the O-0 line is close to the observed lines.

The calculated data in the present work supports the experimental results of Beiersdorfer et al. and suggests that their calculated data is not accurate enough, at least for this transition line. It is accepted in the present work that the O-0 line is weak as compared to the neighboring transitions. The transition energy $\Delta E$ equals $8402.2 \mathrm{eV}$, the weighted oscillator strength $g h_{i j}$ equals $2.05 \times 10^{-3}$ and $A_{r}$ equals $1.26 \times 10^{12} \mathrm{~s}^{-1}$ which makes it very difficult to be experimentally identified as it would be obscured by stronger nearby transitions.

Beiersdorfer et al. identified the O-2 transition line for which $\Delta E=8599.4 \pm 1 \mathrm{eV}$ and its corresponding wavelength $1.4417 \AA$ as $\left(3 s_{1 / 2}\right)_{J=1}-\left(2 p_{3 / 2}\right)_{J=1} \quad[43]$. In the present work, four strong transitions were identified for the same transition line (O-2). They are: (i) $\left(3 s_{1 / 2}\right)_{J=2}-\left(2 p_{3 / 2}\right)_{J=2}$ with $\Delta E=8597.91 \mathrm{eV}$, $g h_{i j}=0.1702$, and $A_{r}=1.82 \times 10^{14} \mathrm{~s}^{-1}$, (ii) $\left(3 s_{1 / 2}\right)_{J=2}-\left(2 p_{1 / 2}\right)_{J=2}$ with $\Delta E=8597.05 \mathrm{eV}$, $g h_{i j}=0.240$, and $A_{r}=1.55 \times 10^{14} \mathrm{~s}^{-1}$, (iii) $\left(3 s_{1 / 2}\right)_{J=0^{-}}\left(2 p_{3 / 2}\right)_{J=1}$ with $\Delta E=8596.48$ $\mathrm{eV}, g h_{i j}=0.113$ and $A_{r}=1.21 \times 10^{14} \mathrm{~s}^{-1}$, and (iv) $\left(3 d_{5 / 2}\right)_{J=0}-\left(2 p_{3 / 2}\right)_{J=1}$ with $\Delta E=8596.29 \mathrm{eV}$, $g f_{i j}=0.323$ and $A_{r}=2.08 \times 10^{14} \mathrm{~s}^{-1}$. Corresponding wavelengths of these four transitions are respectively: $1.4419,1.4420,1.4422$, and $1.4422 \AA$. This suggests that these transitions exhibit blending that makes it difficult to assign each line to a single transition experimentally. More examples of lines blending can be identified in Table II.

Calculated transition energies of optically allowed transitions presented herein compare well with experimental data. The optically allowed strong transitions are grouped into: $n d-2 p, n s-2 p$ and $n p-2 s$ transitions, where $n d-2 p$ is the dominant. The strongest transition line is found to be $3 d_{5 / 2}-2 p_{3 / 2}$ at wavelength $\lambda=1.324 \AA$. Some transition lines in $\mathrm{W}^{66+}$ undergo blending which makes it difficult to assign the experimental features to a specific transition line. In many cases, an experimental line intensifies when it relates to more than one transition.

\subsection{Forbidden transitions of O-like $\mathrm{W}$}

Forbidden transition lines are important in plasma diagnostic. Table SIII in [42] presents the strong forbidden transitions atomic data to the ground state of O-like $\mathrm{W}$ for the electric quadrupole $E_{2}$, magnetic dipole $M_{1}$ and magnetic quadrupole $M_{2}$, respectively. The presented data includes transition energies $\Delta E(\mathrm{eV})$, transition wavelengths $\lambda(\AA)$, radiative transition rates $A_{r}\left(\mathrm{~s}^{-1}\right)$ and weighted oscillator strengths $g f_{i j}$. The ratio of $A$-values of strong forbidden transition $A_{r}$ to $A$-values of electric dipole transitions is found to be of order $10^{-3}$ for $A_{r}\left(E_{2}\right) / A_{r}\left(E_{1}\right)$ and of order $10^{-5}-10^{-4}$ for $A_{r}\left(M_{1}\right) / A_{r}\left(E_{1}\right)$ and $A_{r}\left(M_{2}\right) / A_{r}\left(E_{1}\right)$. The weighted oscillator strength $f$-value ratios are of the orders $g f_{i j}\left(E_{2}\right) / g f_{i j}\left(E_{1}\right) \sim 10^{-3}, g f_{i j}\left(M_{1}\right) / g f_{i j}\left(E_{1}\right)$ and $g f_{i j}\left(M_{2}\right) / g f_{i j}\left(E_{1}\right) \sim 10^{-5}$. The spectral ranges for the forbidden transitions are $\approx 0.8-1.2 \AA, 0.9-1.4 \AA$ and $0.8-1.4 \AA$ for $E_{2}, M_{1}$ and $M_{2}$, respectively. To the best of the author's knowledge, the forbidden transition data for O-like tungsten has not been published anywhere which makes the data presented in this paper of particular importance in experimental plasma diagnostics. 


\subsection{Calculated synthetic spectra of O-like tungsten}

A Doppler line profile with low energy is employed to construct synthetic spectra based on the produced atomic data for electric multipole transitions $E_{1}, E_{2}, M_{1}$ and $M_{2}$ (see Fig. 2) in order to simulate the spectra of low-density tungsten plasma (electron density $n e \leq 10^{13} \mathrm{~cm}^{-1}$ ), where most ions are in ground states, thus making electrons excitation and de-excitation the main spectra generation processes. Constructed synthetic spectra for low-density tungsten plasma are helpful in the diagnostic and identification of spectral features of hotdense plasma, whose synthetic spectra are favorably calculated by collisional-radiative models. Line intensity is normalized to unity in arbitrary units. Figure 2a illustrates the spectrum of optically allowed (electric dipole $E_{1}$ ) transitions of $\mathrm{W}^{66+}$. The strong spectral lines and their transition groups $(2-3,2-4,2-5$ or $2-6)$ are identified and labeled. It is evident that transitions $3 d-2 p$ dominate the spectrum. Theoretical spectra of forbidden transitions: $E_{2}, M_{1}$ and $M_{2}$ are depicted in Fig. 2c and d. It is evident that the spectral ranges of these transitions are comparable. Important forbidden transitions are situated in the allowed transitions range. These synthetic spectra are "fingerprints" of the ion structure of O-like tungsten, because locations of transition lines remain unchanged in a hot dense plasma spectrum while intensities are subject to change due to contributions of other electronic process.

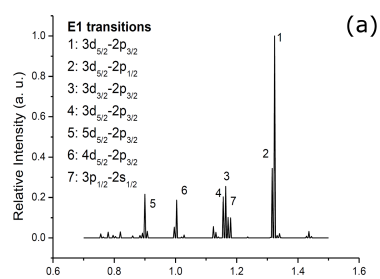

a)
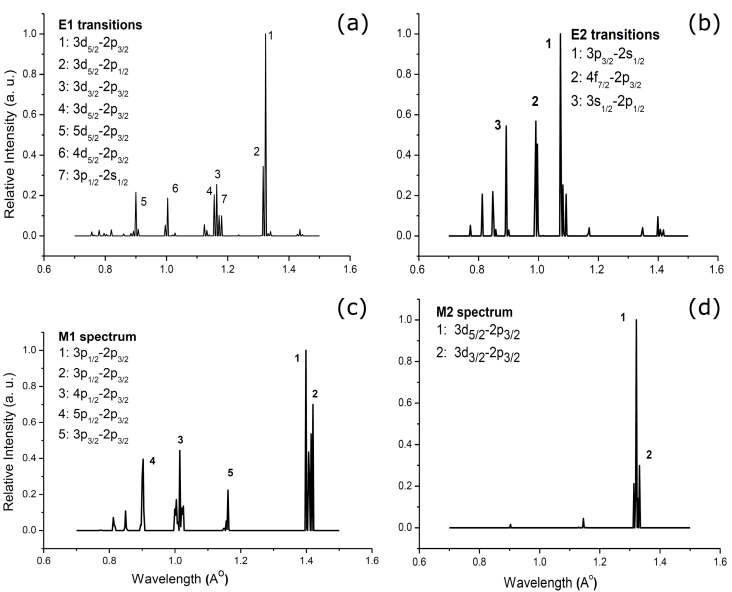

Fig. 2. Theoretical spectrum of: dipole transitions $E_{1}$ of $\mathrm{W}^{66+}$, (a) electric(b) electricdipole transitions $M_{1}$ of $\mathrm{W}^{66+}$, quadrupole transitions $M_{2}$ of $\mathrm{W}^{66+}$.

(c) magnetic-

(d) magnetic-

\section{Summary and conclusions}

Accurate atomic data related to highly ionized tungsten is important in plasma and fusion research. Several tungsten ions received considerable attention in experimental and theoretical research but O-like tungsten $\left(\mathrm{W}^{66+}\right)$ has not attracted enough attention. The relativistic configuration interaction method includes correlation and relativistic effects to all orders and the multi-configuration expansion is applied in this research to carry out atomic structure and spectra calculations for $\mathrm{W}^{66+}$ by two methods: the relativistic configuration interaction method of the flexible atomic code and the multireference many-body perturbation theory (MR-MBPT) approach. The MR-MBPT includes QED effects for high orders which makes it advantageous over the relativistic configuration method of the FAC.

The produced atomic structure and spectral data included energy levels, wavelengths, transition rates and oscillator strengths for allowed and forbidden transitions. The differences in the calculated energy levels by the two methods are very small which suggests that the high orders of QED effects do not have significant contributions to the atomic structure of O-like tungsten. The produced data for allowed and forbidden transitions is used to produce theoretical spectra for O-like $\mathrm{W}$ ion considering the Doppler line profile. The analysis of the synthetic spectra of electric dipole transitions revealed the following:

- the spectral range is about $0.75-1.44 \AA$,

- transitions with $n d 2 p$ are the most important transitions,

- $3 d-2 p$ transitions dominate the spectrum of electrical dipole transitions $E_{1}$,

- $E 2, M_{1}, M_{2}$ transitions are situated in the spectral range of $E_{1}$ transitions.

To the best of the author's knowledge, the present work's results are accurate and form a complete database for O-like tungsten.

\section{Acknowledgments}

The author is grateful to the Hashemite University for granting her a paid sabbatical leave for the 2018/2019 academic year, facilitating her research at Taibah University (Saudi Arabia) which led to the production of atomic data used in this paper.

\section{References}

[1] G.A. Wurden, B.J. Peterson, Rev. Sci. Instrum. 70, 255 (1999).

[2] S. Cowell, A. Hayes, Nuclear Weapons Program Highlights, T16, 2007.

[3] T. Pütterich, R. Neu, R. Dux, A.D. Whiteford, M.G. O'Mullane, Plasma Phys. Control. Fusion 50, 085016 (2008).

[4] P.G. Burkhalter, C.M. Dozier, D.J. Nagel, Phys. Rev. A 15, 700 (1977). 
[5] A. Zigler, H. Zmora, N. Spector, M. Klapish, J.L. Schwob, A.J. BarShalom, J. Opt. Soc. Am. 70, 129 (1980).

[6] P. Mandelbaum, M. Klapisch, A. BarShalom, J. Schwob, A. Zigler, Phys. Lett. A 99, A(2-3) (1983).

[7] M. Klapisch, P. Mandelbaum, A. Barshalom, J.L. Schwob, J. Opt. Soc. Am. 71, 1276 (1981).

[8] S.R. Elliott, P. Beiersdorfer, B.J. McGowan, J. Nilsen, Phys. Rev. A 52, 2689 (1995).

[9] S.R. Elliott, Nucl. Instrum. Methods Phys. Res. B 98, 114 (1995).

[10] A.S. Shlyaptseva, S. Hamasha, S. Hansen, C. Harris, V.L. Kantsyrev, P. Neil, N. Ouart, P. Beiersdorfer, U. Safronova, Rev. Sci. Instrum. 74, 1947 (2003).

[11] A.S. Shlyaptseva, D. Fedin, S. Hamasha et al., Rev. Sci. Instrum. 75, 3750 (2004).

[12] P. Neil, C. Harris, A.S. Safronova, S. Hamasha, S. Hansen, U.I. Safronova, P. Beiersdorfer, Can. J. Phys. 82, 931 (2004).

[13] S.B. Utter, P. Beiersdorfer, E. Träbert, Can. J. Phys. 80, 1503 (2002).

[14] J. Clementson, P. Beiersdorfer, E.W. Magee, H.S. McLean, R.D. Wood, J. Phys. B At. Mol. Opt. Phys. 43, 144009 (2010).

[15] V. Jonauskas, A. Kynienè, P. Rynkun, S. Kučas, G. Gaigalas, R. Kisielius, Š. Masys, G. Merkelis, L. Radžiūtè, J. Phys. B At. Mol. Opt. Phys. 48, 135003 (2015).

[16] X. Ding, J. Liu, F. Koike, I. Murakami, D. Kato, Hiroyuki, A. Sakaue, N. Nakamura, Ch. Dong, Phys. Lett. A 380, 874 (2016).

[17] R. Neu, K.B. Fournier, D. Schlogl, J. Rice, J. Phys. B At. Mol. Phys. 30, 5057 (1997).

[18] K. Asmussen, K.B. Fournier, J.M. Laming, R. Neu, J.F. Seely, R. Dux, W. Engelhardt, J.C. Fuchs and ASDEX Upgrade Team, Nucl. Fusion 38, 967 (1998).

[19] U. Feldman, J.F. Seely, E. Landi, Yu. Ralchenko, Nucl. Fusion 48, 045004 (2008).

[20] J. Yanagibayashi, T. Nakano, A. Iwamae, H. Kubo, M. Hasuo, K. Itami, J. Phys. B At. Mol. Opt. Phys. 43, 144013 (2010).

[21] X.-B. Ding, F. Koike, I. Murakami, D. Kato, H.A. Sakaue, C.-Z. Dong, N. Nakamura, A. Komatsu, J. Sakoda, J. Phys. B At. Mol. Opt. Phys. 44, 145004 (2011).
[22] K.B. Fournier, At. Data Nucl. Data Tables 68, 1 (1998).

[23] Y. Ralchenko, J. Reader, J.M. Pomeroy, J.N. Tan, J.D. Gillaspy, J. Phys. B: At. Mol. Opt. Phys. 40, 3891 (2007).

[24] Y. Ralchenko, J.N. Tan, J.D. Gillaspy, J.M. Pomeroy, Phys. Rev. A 74, 042514 (2006).

[25] L. Özdemir, G.G. Konan, S. Kabakci, Acta Phys. Pol. A 124, 649 (2013).

[26] S. Aggarwal, A.K.S. Jha, M. Mohan, Can. J. Phys. 91, 394 (2012).

[27] S. Aggarwal, Chin. Phys. B 23, 093203 (2014).

[28] R. Radtke, C. Biedermann, P. Mandelbaum, J.L. Schwob, J. Phys. Conf. Series 58, 113 (2007).

[29] A.E. Kramida, T. Shirai, At. Data Nucl. Data Tables 95, 305 (2009).

[30] Z. Fei, R. Zhao, Z. Shi et al., Phys. Rev. A 86, 062501 (2012).

[31] K.M. Aggarwal, F.P. Keenan, Can. J. Phys. 92, 1166 (2014).

[32] P. Quinet, J. Phys. B At. Mol. Opt. Phys. 44, 195007 (2011).

[33] S. Morita, M. Goto, R. Katai, C. Dong, Plasma Sci. Technol. 12, 3 (2010).

[34] M.F. Gu, Can. J. Phys. 86, 675 (2008).

[35] M.F. Gu, T. Holczer, E. Behar, S.M. Kahn, Astrophys. J. 41, 1227 (2006).

[36] M.J. Vilkas, Y. Ishikawa, Phys. Rev. A 72, 032512 (2005).

[37] J. Clementson, P. Beiersdorfer, G.V. Brown, M.F. Gu, Phys. Scr. 81, 015301 (2010).

[38] J. Clementson, P. Beiersdorfer, G.V. Brown, M.F. Gu, H. Lundberg, Y. Podplay, E. Träbert, Can. J. Phys. 89, 571 (2011).

[39] I.J. Lindgren, Physica B 7, 244 (1974).

[40] G. Racah, Phys. Rev. 62, 438 (1942).

[41] S.N. Nahar, W. Eissner, C. Sur, A.K. Pradhan, Phys. Scr. 79, 03540 (2009).

[42] S. Hamasha, Acta Phys. Pol. A 138 , 770.S1 (2020), the supplementary material with Tables SI-SIII, available online only.

[43] P. Beiersdorfer, J.K. Lepson, M.B. Schneider, M.P. Bode, Phys. Rev. A 86, 12509 (2012).

[44] Dipti, Tapasi Das, Lalita Sharma, Rajesh Srivastava, Phys. Scr. 89, 085403 (2014).

[45] F.A. Parpia, C.F. Fischer, P. Grant, Comput. Phys. Commun. 94, 249 (1996).

[46] M.H. Chen, B. Crasemann, Phys. Rev. A 40, 4330 (1989). 